\title{
Einstein-Euler-Heisenberg theory and charged black holes
}

\author{
Remo Ruffini $^{a, b, 41}$, Yuan-Bin Wu ${ }^{a, b, 42}$, She-Sheng Xue ${ }^{a, b} 3$ \\ ${ }^{a}$ Dipartimento di Fisica and ICRA, Sapienza Università di Roma \\ P.le Aldo Moro 5, I-00185 Rome, Italy \\ ${ }^{b}$ ICRANet, P.zza della Repubblica 10, I-65122 Pescara, Italy \\ ${ }^{c}$ ICRANet, University of Nice-Sophia Antipolis, 28 Av. de Valrose, \\ 06103 Nice Cedex 2, France
}

\begin{abstract}
Taking into account the Euler-Heisenberg effective Lagrangian of one-loop nonperturbative quantum electrodynamics (QED) contributions, we formulate the Einstein-Euler-Heisenberg theory and study the solutions of nonrotating black holes with electric and magnetic charges in spherical geometry. In the limit of strong and weak electromagnetic fields of black holes, we calculate the black hole horizon radius, area, and total energy up to the leading order of QED corrections and discuss the black hole irreducible mass, entropy, and maximally extractable energy as well as the Christodoulou-Ruffini mass formula. We find that these black hole quantities receive the QED corrections, in comparison with their counterparts in the Reissner-Nordström solution. The QED corrections show the screening effect on black hole electric charges and the paramagnetic effect on black hole magnetic charges. As a result, the black hole horizon area, irreducible mass, and entropy increase; however, the black hole total energy and maximally extractable energy decrease, compared with the Reissner-Nordström solution. In addition, we show that the condition for extremely charged black holes is modified due to the QED correction.
\end{abstract}

PACS: 12.20.-m, 13.40-f, 11.27.+d, 04.70.-s

Keywords: Quantum Electrodynamics, Black Holes, Euler-Heisenberg effective Lagrangian

${ }^{1}$ ruffini@icra.it

${ }^{2}$ Corresponding author.wuyb@icranet.org

${ }^{3}$ xue@icra.it 


\section{Introduction}

For several decades the nonlinear electromagnetic generalization of the Reissner-Nordström solution of the Einstein-Maxwell equations has attracted a great deal of attention. The most popular example is the gravitating Born-Infeld (BI) theory [1]. The static charged black holes in gravitating nonlinear electrodynamics were studied in the 1930s [2, 3]. The discovery that the string theory, as well as the D-brane physics, leads to Abelian and non-Abelian BI-like Lagrangians in its low-energy limit (see, e.g., Refs. [4-6]), has renewed the interest in these kinds of nonlinear actions. Asymptotically flat, static, spherically symmetric black hole solutions for the Einstein-Born-Infeld theory were obtained in the literature [7, 8].

Generalization of the exact solutions of spherically symmetric Born-Infeld black holes with a cosmological constant in arbitrary dimensions has been considered [9-11], as well as in other gravitational backgrounds [12,13]. Many other models of nonlinear electrodynamics leading to static and spherically symmetric structures have been considered in the last decades, such as the theory with a nonlinear Lagrangian of a general function of the gauge invariants $\left(F^{\mu v} F_{\mu \nu}\right.$ and $\left.F_{\mu v} \tilde{F}^{\mu v}\right)$ [14-17] or a logarithmic function of the Maxwell invariant $\left(F^{\mu v} F_{\mu v}\right)$ [18], and the theory with a generalized nonlinear Lagrangian [19] which can lead to the BI Lagrangian and the weak-field limit of the Euler-Heisenberg effective Lagrangian [20]. The static and spherically symmetric black hole, whose gravity coupled to the nonlinear electrodynamics of the weakfield limit of the Euler-Heisenberg effective Lagrangian as a low-energy limit of the Born-Infeld theory, was studied in Ref. [21]. Some attempts in the obtention of regular (singularity-free) static and spherically symmetric black hole solutions in gravitating nonlinear electrodynamics have been made [22-26], and the unusual properties of these solutions have been discussed in Refs. [27, 28]. Generalization of spherically symmetric black holes in higher dimension in the theory with a nonlinear Lagrangian of a function of power of the Maxwell invariant has been considered in the literature [29-32]. Finally, we mention that rotating black branes [33, 34] and rotating black strings [35] in the Einstein-Born-Infeld theory have been also considered.

The effective Lagrangian of nonlinear electromagnetic fields has been formulated for the first time by Heisenberg and Euler using the Dirac electron-positron theory [20]. Schwinger reformulated this nonperturbative one-loop effective Lagrangian within the quantum electrodynamics (QED) framework [36]. This effective Lagrangian characterizes the phenomenon of vacuum polarization. Its imaginary part describes the probability of the vacuum decay via the electron-positron pair production. If electric fields are stronger than the critical value $E_{c}=m^{2} c^{3} / e \hbar$, the energy of the vacuum can be lowered by spontaneously creating electronpositron pairs [20, 36, 37]. For many decades, both theorists and experimentalists have been interested in the aspects of the electron-positron pair production from the QED vacuum and the vacuum polarization by an external electromagnetic field (see, e.g., Refs. [38, 39]).

As a fundamental theory, QED gives an elegant description of the electromagnetic interaction; moreover, it has been experimentally verified. Therefore, it is important to study the QED effects in black hole physics. As a result of one-loop nonperturbative QED, the EulerHeisenberg effective Lagrangian deserves to attract more attention in the topic of generalized 
black hole solutions mentioned above. In this article, we adopt the contribution from the EulerHeisenberg effective Lagrangian to formulate the Einstein-Euler-Heisenberg theory, and study the solutions of electrically and magnetically charged black holes in spherical geometry. We calculate and discuss the QED corrections to the black hole horizon area, entropy, total energy, and the maximally extractable energy.

The article is organized as follows. In Sec. 2, we first recall the Euler-Heisenberg effective Lagrangian. We formulate the Einstein-Euler-Heisenberg theory in Sec. 3. The study of electrically charged black holes in the weak electric field case is presented in Sec. 4. The study of magnetically charged black holes in both weak and strong magnetic field cases is presented in Sec. 5. Then we present the study of black holes with both electric and magnetic charges in the Einstein-Euler-Heisenberg theory in Sec.6 A summary is given in Sec. 7 The use of units with $\hbar=c=1$ is throughout the article.

\section{The Euler-Heisenberg effective Lagrangian}

The QED one-loop effective Lagrangian was obtained by Heisenberg and Euler [20] for constant electromagnetic fields,

$$
\begin{aligned}
\Delta \mathscr{L}_{\mathrm{eff}}= & \frac{1}{2(2 \pi)^{2}} \int_{0}^{\infty} \frac{d s}{s^{3}}\left[e^{2} \varepsilon \beta s^{2} \operatorname{coth}(e \varepsilon s) \cot (e \beta s)\right. \\
& \left.-1-\frac{e^{2}}{3}\left(\varepsilon^{2}-\beta^{2}\right) s^{2}\right] e^{-i s\left(m_{e}^{2}-i \eta\right)}
\end{aligned}
$$

as a function of two invariants: the scalar $S$ and the pseudoscalar $P$,

$$
\begin{aligned}
S & \equiv-\frac{1}{4} F_{\mu \nu} F^{\mu \nu}=\frac{1}{2}\left(\mathbf{E}^{2}-\mathbf{B}^{2}\right) \equiv \varepsilon^{2}-\beta^{2}, \\
P & \equiv-\frac{1}{4} F_{\mu \nu} \tilde{F}^{\mu \nu}=\mathbf{E} \cdot \mathbf{B} \equiv \varepsilon \beta,
\end{aligned}
$$

where the field strength is $F^{\mu \nu}, \tilde{F}^{\mu \nu} \equiv \varepsilon^{\mu \nu \lambda \kappa} F_{\lambda \kappa} / 2$, and

$$
\begin{aligned}
\varepsilon & =\sqrt{\left(S^{2}+P^{2}\right)^{1 / 2}+S} \\
\beta & =\sqrt{\left(S^{2}+P^{2}\right)^{1 / 2}-S} .
\end{aligned}
$$

The effective Lagrangian reads

$$
\mathscr{L}_{\text {eff }}=\mathscr{L}_{M}+\Delta \mathscr{L}_{\text {eff }}
$$

where $\mathscr{L}_{M}=S$ is the Maxwell Lagrangian. Its imaginary part is related to the decay rate of the vacuum per unit volume [20, 36],

$$
\frac{\Gamma}{V}=\frac{\alpha \varepsilon^{2}}{\pi^{2}} \sum_{n=1} \frac{1}{n^{2}} \frac{n \pi \beta / \varepsilon}{\tanh n \pi \beta / \varepsilon} \exp \left(-\frac{n \pi E_{c}}{\varepsilon}\right)
$$


for fermionic fields, and

$$
\frac{\Gamma}{V}=\frac{\alpha \varepsilon^{2}}{2 \pi^{2}} \sum_{n=1} \frac{(-1)^{n}}{n^{2}} \frac{n \pi \beta / \varepsilon}{\sinh n \pi \beta / \varepsilon} \exp \left(-\frac{n \pi E_{c}}{\varepsilon}\right)
$$

for bosonic fields; here, $E_{c}=\frac{m_{e}^{2} c^{3}}{e \hbar}$ is the critical field. Using the expressions [40]

$$
\begin{array}{ll}
e \varepsilon s \operatorname{coth}(e \varepsilon s)=\sum_{n=-\infty}^{\infty} \frac{s^{2}}{\left(s^{2}+\tau_{n}^{2}\right)}, & \tau_{n} \equiv n \pi / e \varepsilon \\
e \beta s \cot (e \beta s)=\sum_{m=-\infty}^{\infty} \frac{s^{2}}{\left(s^{2}-\tau_{m}^{2}\right)}, & \tau_{m} \equiv m \pi / e \beta
\end{array}
$$

one obtains the real part of the Euler-Heisenberg effective Lagrangian (1) (see Refs. [38, 4145]),

$$
\begin{aligned}
\left(\Delta \mathscr{L}_{\mathrm{eff}}^{\mathrm{cos}}\right)_{\mathscr{P}} & =\frac{1}{2(2 \pi)^{2}} \sum_{n, m=-\infty}^{\infty} \frac{1}{\tau_{m}^{2}+\tau_{n}^{2}}\left[\bar{\delta}_{m 0} J\left(i \tau_{m} m_{e}^{2}\right)-\bar{\delta}_{n 0} J\left(\tau_{n} m_{e}^{2}\right)\right] \\
& =-\frac{1}{(2 \pi)^{2}}\left[\sum_{n=1}^{\infty} \frac{e \beta}{\tau_{n}} \operatorname{coth}\left(e \beta \tau_{n}\right) J\left(\tau_{n} m_{e}^{2}\right)-\sum_{m=1}^{\infty} \frac{e \varepsilon}{\tau_{m}} \operatorname{coth}\left(e \varepsilon \tau_{m}\right) J\left(i \tau_{m} m_{e}^{2}\right)\right]
\end{aligned}
$$

The symbol $\bar{\delta}_{i j} \equiv 1-\delta_{i j}$ denotes the complimentary Kronecker $\delta$, which vanishes for $i=j$, and

$$
J(z) \equiv \mathscr{P} \int_{0}^{\infty} d s \frac{s e^{-s}}{s^{2}-z^{2}}=-\frac{1}{2}\left[e^{-z} \operatorname{Ei}(z)+e^{z} \operatorname{Ei}(-z)\right] .
$$

Here, $\mathscr{P}$ indicates the principle value integral, and $\operatorname{Ei}(z)$ is the exponential-integral function,

$$
\operatorname{Ei}(z) \equiv \mathscr{P} \int_{-\infty}^{z} d t \frac{e^{t}}{t}=\log (-z)+\sum_{k=1}^{\infty} \frac{z^{k}}{k k !}
$$

Using the series and asymptotic representation of the exponential-integral function $\operatorname{Ei}(z)$ for large $z$ corresponding to weak electromagnetic fields $\left(\varepsilon / E_{c} \ll 1, \beta / E_{c} \ll 1\right)$,

$$
J(z)=-\frac{1}{z^{2}}-\frac{6}{z^{4}}-\frac{120}{z^{6}}-\frac{5040}{z^{8}}-\frac{362880}{z^{10}}+\cdots,
$$

the weak-field expansion of Eq. (10) is

$$
\left(\Delta \mathscr{L}_{\text {eff }}\right)_{\mathscr{P}}=\frac{2 \alpha^{2}}{45 m_{e}^{4}}\left\{4 S^{2}+7 P^{2}\right\}+\frac{64 \pi \alpha^{3}}{315 m_{e}^{8}}\left\{16 S^{3}+26 S P^{2}\right\}+\cdots,
$$

which is expressed in terms of a powers series of weak electromagnetic fields up to $O\left(\alpha^{3}\right)$, the first term was obtained by Heisenberg and Euler in their original article [20].

On the other hand, using the series and asymptotic representation of the exponential-integral function $\operatorname{Ei}(z)$ for small $z \ll 1$ [40] corresponding to strong electromagnetic fields $\left(\varepsilon / E_{c} \gg 1\right.$, $\left.\beta / E_{c} \gg 1\right)$,

$$
J(z)=-\frac{1}{2}\left[e^{z} \ln (z)+e^{-z} \ln (-z)\right]-\frac{1}{2} \gamma\left[e^{z}+e^{-z}\right]+\mathscr{O}(z)
$$


the leading terms in the strong-field expansion of Eqs. (10) and (11) are given by (see Refs. [38, 41, 45, 46])

$$
\begin{aligned}
& \left(\Delta \mathscr{L}_{\mathrm{eff}}^{\cos }\right)_{\mathscr{P}}=\frac{1}{2(2 \pi)^{2}} \sum_{n, m=-\infty}^{\infty} \frac{1}{\tau_{m}^{2}+\tau_{n}^{2}}\left[\bar{\delta}_{n 0} \ln \left(\tau_{n} m_{e}^{2}\right)-\bar{\delta}_{m 0} \ln \left(\tau_{m} m_{e}^{2}\right)\right]+\cdots \\
& =\frac{1}{2(2 \pi)^{2}}\left[\sum_{n=1}^{\infty} \frac{e \beta}{\tau_{n}} \operatorname{coth}\left(e \beta \tau_{n}\right) \ln \left(\tau_{n} m_{e}^{2}\right)-\sum_{m=1}^{\infty} \frac{e \varepsilon}{\tau_{m}} \operatorname{coth}\left(e \varepsilon \tau_{m}\right) \ln \left(\tau_{m} m_{e}^{2}\right)\right]+\cdots
\end{aligned}
$$

In the case of vanishing magnetic field $\mathbf{B}=0$ and a strong electric field $E \gg E_{c}$ using $\lim _{z \rightarrow \infty} J(i z)=$ 0 and $\lim _{z \rightarrow 0} z \operatorname{coth}(a z)=1 / a$, Eq. (18) becomes (see Refs. [38,41,45])

$$
\begin{aligned}
\left(\Delta \mathscr{L}_{\mathrm{eff}}^{\mathrm{cos}}\right)_{\mathscr{P}} & =\frac{e^{2} E^{2}}{4 \pi^{4}} \sum_{n=1}^{\infty} \frac{1}{n^{2}}\left[\ln \left(\frac{n \pi E_{c}}{E}\right)+\gamma\right]+\cdots \\
& =\frac{e^{2} E^{2}}{24 \pi^{2}}\left[\ln \left(\frac{\pi E_{c}}{E}\right)+\gamma\right]-\frac{e^{2} E^{2}}{4 \pi^{4}} \zeta^{\prime}(2)+\cdots,
\end{aligned}
$$

with the Euler-Mascheroni constant $\gamma=0.577216$, the Riemann zeta function $\zeta(k)=\sum_{n} 1 / n^{k}$, and

$$
\zeta^{\prime}(2)=\frac{\pi^{2}}{6}[\gamma+\ln (2 \pi)-12 \ln A] \simeq-0.937548,
$$

with $A=1.28243$ being the Glaisher constant. Similarly, in the case of vanishing electric field $\mathbf{E}=0$ and a strong magnetic field $B \gg E_{c}$, Eq. (18) becomes (see Refs. [38, 41, 45])

$$
\begin{aligned}
\left(\Delta \mathscr{L}_{\mathrm{eff}}^{\mathrm{cos}}\right)_{\mathscr{P}} & =-\frac{e^{2} B^{2}}{4 \pi^{4}} \sum_{m=1}^{\infty} \frac{1}{n^{2}}\left[\ln \left(\frac{n \pi E_{c}}{B}\right)+\gamma\right]+\cdots \\
& =-\frac{e^{2} B^{2}}{24 \pi^{2}}\left[\ln \left(\frac{\pi E_{c}}{B}\right)+\gamma\right]+\frac{e^{2} B^{2}}{4 \pi^{4}} \zeta^{\prime}(2)+\cdots
\end{aligned}
$$

The $(n=1)$ term in Eq. (22) is the one obtained by Weisskopf [47].

\section{The Einstein-Euler-Heisenberg theory}

Since the real part of the Euler-Heisenberg effective Lagrangian $\left(\Delta \mathscr{L}_{\mathrm{eff}}^{\text {cos }}\right) \mathscr{P}$ of Eq. (10) is expressed in terms of Lorentz invariants $(\varepsilon, \beta)$ or $(S, P)$, the Euler-Heisenberg effective action in the curve space-time described by metric $g_{\mu \nu}$ can be written as

$$
\mathscr{S}_{\mathrm{EH}}=\int d^{4} x \sqrt{-g} \mathscr{L}_{\mathrm{EH}}, \quad \mathscr{L}_{\mathrm{EH}}=\left[S+\left(\Delta \mathscr{L}_{\mathrm{eff}}^{\mathrm{cos}}\right) \mathscr{P}\right]
$$

The Einstein and Euler-Heisenberg action is then given by

$$
\mathscr{S}_{\mathrm{EEH}}=-\frac{1}{16 \pi G} \int d^{4} x \sqrt{-g} R+\mathscr{S}_{\mathrm{EH}}
$$

where $R$ is the Ricci scalar. 
The Einstein field equations are

$$
G^{\mu v} \equiv R^{\mu v}-\frac{1}{2} g^{\mu v} R=8 \pi G T^{\mu v}
$$

where the energy-momentum tensor is

$$
T^{\mu v}=\frac{2}{\sqrt{-g}} \frac{\delta \mathscr{S}_{\mathrm{EH}}}{\delta g_{\mu v}} .
$$

The electromagnetic field equations and Bianchi identities are given by

$$
\mathscr{D}_{\mu} P^{v \mu}=j^{v}, \quad \mathscr{D}_{\mu} \tilde{F}^{\mu v}=0
$$

and the displacement fields $P^{v \mu}, D^{i}=P^{0 i}$, and $H^{i}=-\varepsilon^{i j k} P_{j k}$ are defined as

$$
P^{\mu v}=\frac{\delta \mathscr{L}_{\mathrm{EH}}}{\delta F_{\mu \nu}}, \quad D^{i}=\frac{\delta \mathscr{L}_{\mathrm{EH}}}{\delta E_{i}}, \quad H^{i}=-\frac{\delta \mathscr{L}_{\mathrm{EH}}}{\delta B_{i}} .
$$

Here, electromagnetic fields are treated as smooth varying fields over all space generated by external charge currents $j^{\mu}$ at infinity.

Using functional derivatives, we obtain

$$
\begin{aligned}
T^{\mu v} & =-g^{\mu v}\left[S+\left(\Delta \mathscr{L}_{\mathrm{eff}}^{\mathrm{cos}}\right) \mathscr{P}\right]+2\left[\frac{\delta S}{\delta g_{\mu \nu}} \frac{\delta \mathscr{L}_{\mathrm{EH}}}{\delta S}+\frac{\delta P}{\delta g_{\mu \nu}} \frac{\delta \mathscr{L}_{\mathrm{EH}}}{\delta P}\right], \\
& =-g^{\mu v}\left[S+\left(\Delta \mathscr{L}_{\mathrm{eff}}^{\mathrm{cos}}\right) \mathscr{P}\right]+2\left[\left(1+\mathscr{A}_{S}\right) \frac{\delta S}{\delta g_{\mu v}}+\mathscr{A}_{P} \frac{\delta P}{\delta g_{\mu v}}\right],
\end{aligned}
$$

where two invariants are defined as

$$
\mathscr{A}_{S} \equiv \frac{\delta\left(\Delta \mathscr{L}_{\mathrm{eff}}^{\mathrm{cos}}\right) \mathscr{P}}{\delta S} ; \quad \mathscr{A}_{P} \equiv \frac{\delta\left(\Delta \mathscr{L}_{\mathrm{eff}}^{\mathrm{cos}}\right) \mathscr{P}}{\delta P} .
$$

It is straightforward to obtain

$$
\frac{\delta S}{\delta g_{\mu v}}=\frac{1}{2} F_{\lambda}^{\mu} F^{\lambda v}, \quad \frac{\delta P}{\delta g_{\mu v}}=F_{\lambda}^{\mu} \tilde{F}^{\lambda v}=g^{\mu v} P,
$$

and as a result, we rewrite Eq. (30) as

$$
\begin{aligned}
T^{\mu v} & =T_{M}^{\mu v}+g^{\mu v}\left[\mathscr{A}_{P} P-\left(\Delta \mathscr{L}_{\mathrm{eff}}^{\mathrm{cos}}\right) \mathscr{P}\right]+\mathscr{A}_{S} F_{\lambda}^{\mu} F^{\lambda v}, \\
& =T_{M}^{\mu v}\left(1+\mathscr{A}_{S}\right)+g^{\mu v}\left[\mathscr{A}_{S} S+\mathscr{A}_{P} P-\left(\Delta \mathscr{L}_{\mathrm{eff}}^{\mathrm{cos}}\right) \mathscr{P}\right],
\end{aligned}
$$

where $T_{M}^{\mu v}=-g^{\mu v} S+F_{\lambda}^{\mu} F^{\lambda v}$ is the energy-momentum tensor of the electromagnetic fields of the linear Maxwell theory. Equation (33) is in fact a general result, independent of the explicit form of nonlinear Lagrangian $\left(\Delta \mathscr{L}_{\mathrm{eff}}^{\mathrm{cos}}\right) \mathscr{P}$. Equations (24)-(33) in principle give a complete set of equations for Einstein and Euler-Heisenberg effective theory, together with total charge 
$(Q)$, angular-momentum $(L)$, and energy $(M)$ conservations. In this article, adopting the EulerHeisenberg effective Lagrangian (10), we explicitly calculate invariants $\mathscr{A}_{S}$ and $\mathscr{A}_{P}$ of Eq. (31) as well as the energy-momentum $T^{\mu \nu}$ of Eq. (33) in the following cases.

It is necessary to point out that in present article, we do not consider the couplings between photons and gravitons that are also induced by QED vacuum polarization effects at the level of one-fermion loop. Drummond and Hathrell obtained the photon effective action from the lowest term of one-loop vacuum polarization on a general curved background manifold; i.e., a graviton couples to two on-mass-shell photons through a fermionic loop [48],

$$
\begin{aligned}
\mathscr{S}_{\mathrm{DH}}=-\frac{\alpha}{720 \pi m_{e}^{2}} \int d^{4} x \sqrt{-g} & \left(5 R F_{\mu v} F^{\mu v}-26 R_{\mu v} F^{\mu \sigma} F_{\sigma}^{v}\right. \\
& \left.+2 R_{\mu v \sigma \tau} F^{\mu v} F^{\sigma \tau}+24 \mathscr{D}_{\mu} F^{\mu v} \mathscr{D}_{\sigma} F_{v}^{\sigma}\right) .
\end{aligned}
$$

Further studies of one-loop effective action (34) were made based on the approach of the heatkernel or "inverse mass" expansion [49, 50], the approach of the so-called "derivative expansion" [51, 52], and the consideration of the one-loop one particle irreducible of one graviton interacting with any number of photons [53]. This effective action (34) was used to study the modified photon dispersion relation by a generic gravitational background [48] and the possible consequences [54-57].

At the level of one-loop quantum corrections of the QED theory in the presence of gravitational field, the effective Lagrangian (34) should be considered as an addition to the Euler and Heisenberg effective Lagrangian (15) in the weak-field limit. In this article, we try to quantitatively study the QED corrections in spherically symmetric black holes with mass $M$ and charge $Q$. In this case, the corrections from the Euler and Heisenberg effective Lagrangian (15) must be much larger than the one from the effective Lagrangian (34). Studying the discussion and result of Ref. [48] for spherical symmetric black holes, we approximately estimate the ratio of Eqs. (15) and (34) around the horizon of black holes with mass $M$ and charge $Q$. As a result, this ratio is $\sim 10^{-2}\left(\frac{Q}{M \sqrt{G}}\right)^{2} \frac{\alpha}{G m_{e}^{2}} \gg 1$. It is not surprising that the electromagnetic coupling $e \sim 1 / \sqrt{137}$ is much larger than the effective gravitational counterpart $G m_{e}^{2} \sim 10^{-45}$. Besides, it is expected that calculations involving both the Euler-Heisenberg effective Lagrangian (15) and Eq. (34) are much more complex and tedious. Nevertheless, it is interesting to investigate the effect of the photon-graviton amplitudes on black hole physics. In this article, for the sake of simplicity, we first consider only the Einstein-Euler-Heisenberg action (25) as a leading contribution in order to gain some physical insight into the QED corrections in black hole physics.

\section{1 $\mathbf{B}=0, \mathbf{E} \neq 0$ or $\mathbf{E}=0, \mathbf{B} \neq 0$}

We consider the case of $\mathbf{B}=0$ and $\mathbf{E} \neq 0$, namely, $\beta=P=0, \varepsilon=E=|\mathbf{E}|$, and $S=E^{2} / 2$. $\mathscr{A}_{P}=0$ and the effective Lagrangian Eq. (10) becomes

$$
\left(\Delta \mathscr{L}_{\mathrm{eff}}^{\mathrm{cos}}\right)_{\mathscr{P}}=-\frac{e^{2} E^{2}}{4 \pi^{4}} \sum_{n=1}^{\infty} \frac{1}{n^{2}} J\left(n \pi E_{c} / E\right) .
$$


Using

$$
\mathscr{P} \int_{0}^{\infty} d s \frac{e^{-s}}{\left(s^{2}-z^{2}\right)}=-\frac{1}{2 z}\left[e^{-z} \operatorname{Ei}(z)-e^{z} \operatorname{Ei}(-z)\right]
$$

we calculate

$$
\frac{d J(z)}{d z^{2}}=\mathscr{P} \int_{0}^{\infty} d s \frac{s e^{-s}}{\left(s^{2}-z^{2}\right)^{2}}=\frac{1}{2 z^{2}}-\mathscr{P} \int_{0}^{\infty} d s \frac{e^{-s}}{\left(s^{2}-z^{2}\right)}
$$

and obtain

$$
\begin{aligned}
\mathscr{A}_{S}= & -\frac{e^{2}}{2 \pi^{4}} \sum_{n=1}^{\infty} \frac{1}{n^{2}} J\left(n \pi E_{c} / E\right) \\
& -\frac{e^{2}}{4 \pi^{2}} \zeta(2)+\frac{e^{2}}{4 \pi} \frac{E_{c}}{E} \sum_{n=1}^{\infty} \frac{1}{n} \tilde{J}\left(n \pi E_{c} / E\right),
\end{aligned}
$$

where

$$
\tilde{J}(z)=e^{-z} \operatorname{Ei}(z)-e^{z} \operatorname{Ei}(-z) .
$$

Substituting these quantities into Eq. (33), we obtain the expression of the energy-momentum tensor $T^{\mu v}(\varepsilon)$. In the case of $\mathbf{E}=0$ and $\mathbf{B} \neq 0$, the energy-momentum tensor $T^{\mu v}(\beta)$ can be straightforwardly obtained from $T^{\mu v}(\varepsilon)$ by the discrete duality transformation $\varepsilon \rightarrow i \beta$, i.e., $|\mathbf{E}| \rightarrow i|\mathbf{B}|$. In principle, using the complete Euler-Heisenberg effective Lagrangian $\left(\Delta \mathscr{L}_{\text {eff }}^{\text {cos }}\right) \mathscr{P}$ (10) for arbitrary electromagnetic fields $\mathbf{E}$ and $\mathbf{B}$, one can obtain the energy-momentum tensor $T^{\mu v}(\varepsilon, \beta)$ of Eq. (33). For the reason of practical calculations, we consider the cases of weak and strong fields.

\subsection{Weak- and strong-field cases}

In the weak-field case, using Eq. (15) and calculating Eqs. (30)-(33), we obtain

$$
\begin{aligned}
& \mathscr{A}_{S}=\frac{2 \alpha^{2}}{45 m_{e}^{4}}(8 S)+\frac{64 \pi \alpha^{3}}{315 m_{e}^{8}}\left(48 S^{2}+26 P^{2}\right)+\cdots, \\
& \mathscr{A}_{P}=\frac{2 \alpha^{2}}{45 m_{e}^{4}}(14 P)+\frac{64 \pi \alpha^{3}}{315 m_{e}^{8}}(52 S P)+\cdots,
\end{aligned}
$$

and

$$
T^{\mu v}=T_{M}^{\mu v}\left[1+8\left(\frac{2 \alpha^{2}}{45 m_{e}^{4}}\right) S\right]+g^{\mu v}\left(\frac{2 \alpha^{2}}{45 m_{e}^{4}}\right)\left[4 S^{2}+7 P^{2}\right]+\cdots,
$$

up to the leading order.

In strong-field case $\varepsilon / E_{c} \gg 1$ and $\beta / E_{c} \gg 1$ using Eq. (17) and calculating Eqs. (30)-(33), we obtain

$$
\begin{aligned}
\mathscr{A}_{S}= & \frac{1}{2(2 \pi)^{2}} \frac{2}{\varepsilon^{2}+\beta^{2}} \sum_{n, m=-\infty}^{\infty} \frac{1}{\left(\tau_{m}^{2}+\tau_{n}^{2}\right)^{2}}\left\{\bar{\delta}_{n 0}\left[\left(\tau_{n}^{2}-\tau_{m}^{2}\right) \ln \left(\tau_{n} m_{e}^{2}\right)-\frac{1}{2}\left(\tau_{m}^{2}+\tau_{n}^{2}\right)\right]\right. \\
& \left.-\bar{\delta}_{m 0}\left[\left(\tau_{n}^{2}-\tau_{m}^{2}\right) \ln \left(\tau_{m} m_{e}^{2}\right)+\frac{1}{2}\left(\tau_{m}^{2}+\tau_{n}^{2}\right)\right]\right\}+\cdots
\end{aligned}
$$


and

$$
\begin{aligned}
\mathscr{A}_{P}= & \frac{1}{2(2 \pi)^{2}} \frac{2 \varepsilon \beta}{\varepsilon^{2}+\beta^{2}} \sum_{n, m=-\infty}^{\infty} \frac{1}{\left(\tau_{m}^{2}+\tau_{n}^{2}\right)^{2}}\left\{\bar{\delta}_{n 0}\left[\left(\frac{\tau_{n}^{2}}{\varepsilon^{2}}+\frac{\tau_{m}^{2}}{\beta^{2}}\right) \ln \left(\tau_{n} m_{e}^{2}\right)-\frac{1}{2} \frac{\left(\tau_{m}^{2}+\tau_{n}^{2}\right)}{\varepsilon^{2}}\right]\right. \\
& \left.-\bar{\delta}_{m 0}\left[\left(\frac{\tau_{n}^{2}}{\varepsilon^{2}}+\frac{\tau_{m}^{2}}{\beta^{2}}\right) \ln \left(\tau_{m} m_{e}^{2}\right)-\frac{1}{2} \frac{\left(\tau_{m}^{2}+\tau_{n}^{2}\right)}{\beta^{2}}\right]\right\}+\cdots
\end{aligned}
$$

From Eq. (20) for $\mathbf{B}=0$ and a strong electric field, we obtain

$$
\mathscr{A}_{S}=\frac{e^{2}}{24 \pi^{2}}\left[2 \ln \left(\frac{\pi E_{c}}{E}\right)+2 \gamma-1\right]-\frac{e^{2}}{2 \pi^{4}} \zeta^{\prime}(2)+\cdots,
$$

and the energy-momentum tensor $T^{\mu v}$ of Eq. (33),

$$
T^{\mu v}=T_{M}^{\mu \nu}\left\{1+\frac{e^{2}}{24 \pi^{2}}\left[2 \ln \left(\frac{\pi E_{c}}{E}\right)+2 \gamma-1\right]-\frac{e^{2}}{2 \pi^{4}} \zeta^{\prime}(2)\right\}-g^{\mu \nu} \frac{e^{2} E^{2}}{48 \pi^{2}}+\cdots
$$

Analogously, from Eq. (23) for $\mathbf{E}=0$ and a strong magnetic field, we obtain

$$
\mathscr{A}_{S}=\frac{e^{2}}{24 \pi^{2}}\left[2 \ln \left(\frac{\pi E_{c}}{B}\right)+2 \gamma-1\right]-\frac{e^{2}}{2 \pi^{4}} \zeta^{\prime}(2)+\cdots,
$$

and the energy-momentum tensor

$$
T^{\mu v}=T_{M}^{\mu v}\left\{1+\frac{e^{2}}{24 \pi^{2}}\left[2 \ln \left(\frac{\pi E_{c}}{B}\right)+2 \gamma-1\right]-\frac{e^{2}}{2 \pi^{4}} \zeta^{\prime}(2)\right\}+g^{\mu v} \frac{e^{2} B^{2}}{48 \pi^{2}}+\cdots
$$

In the following sections, using the energy-momentum tensors $T^{\mu v}$ of Eqs. (41), (45), and (47), we try to study the solutions of the Einstein-Euler-Heisenberg theory for nonrotating (spherically symmetric), electrically or magnetically charged black holes.

\section{Electrically charged black holes}

In this section, we study a nonrotating (spherically symmetric) electrically charged black hole. In this spherical symmetry case, the gauge potential is

$$
A_{\mu}(x)=\left[A_{0}(r), 0,0,0\right]
$$

corresponding to the electric field $E(r)=-A_{0}^{\prime}(r)=-\partial A_{0}(r) / \partial r$ in the radial direction, and the metric field is assumed to be

$$
d s^{2}=f(r) d t^{2}-f(r)^{-1} d r^{2}-r^{2} d \Omega ; \quad f(r) \equiv 1-2 G m(r) / r .
$$

The metric function $f(r)$ and the electric field $E(r)$ fulfill the Einstein equations (26) and electromagnetic field equations (28) and their asymptotically flat solutions at $r \gg 1$,

$$
A_{0}(r) \rightarrow-\frac{Q}{4 \pi r}, \quad E(r) \rightarrow \frac{Q}{4 \pi r^{2}}, \quad \frac{G m(r)}{r} \rightarrow \frac{G M}{r}
$$


satisfy the Gauss law, where $Q$ and $M$ are the black hole electric charge and mass seen at infinity.

In order to find the solution near to the horizon of the black hole by taking into account the QED effects, we approximately adopt the Euler-Heisenberg effective Lagrangian for constant fields that leads to the energy-momentum tensor (41) or (45) for $\mathbf{B}=0$. This approximation is based on the assumption that the macroscopic electric field $E(r)$ is approximated as a constant field $E$ over the microscopic scale of the electron Compton lengths. When the electric field of charged black holes are overcritical, electron-positron pair productions take place and the electric field is screened down to its critical value $E_{c}$ (see Refs. [58-61]). In this article, we study the QED effects on electrically charged black holes with spherical symmetry, whose electric field is much smaller than the critical field $E_{c}$. In this weak electric field case using Eq. (41) we obtain the energy-momentum tensor

$$
T^{\mu \nu}=T_{M}^{\mu v}\left(1+\frac{2 \alpha E^{2}}{45 \pi E_{c}^{2}}\right)+g^{\mu \nu} \frac{\alpha E^{4}}{90 \pi E_{c}^{2}}+\cdots .
$$

As a result, the (0-0) component of Einstein equations is

$$
\frac{2 m^{\prime}(r)}{r^{2}}=4 \pi\left[E^{2}(r)+\frac{\alpha}{15 \pi} E^{4}(r) / E_{c}^{2}\right],
$$

which relates to the energy conservation. Analogously, using Eqs. (28) and (29) and the metric of Eq. (49), we obtain the field equation up to the leading order,

$$
\frac{2 \alpha}{45 \pi} E^{3}(r) / E_{c}^{2}+E(r)=\frac{Q}{4 \pi r^{2}},
$$

which is the zero component of $\mathscr{D}_{\mu} P^{v \mu}=j^{v}$ of Eq. (28) in the spherical symmetry case. This equation relates to the total charge conservation.

A similar case was studied in Ref. [21], in which, however, the effective Lagrangian [the first term in Eq. [15)] was considered as a low-energy limit of the Born-Infeld theory; the coefficients of the $S^{2}$ and $P^{2}$ terms in Eq. (15) are treated as free parameters, so as to either numerically or analytically study the properties of spherically symmetric black hole solutions in the EinsteinEuler-Heisenberg system. In the following, in order to analytically study the QED effects on the black hole solution, we use the Euler-Heisenberg effective Lagrangian (15) and find the black hole solution by a series expansion in powers of $\alpha$. Introducing $\bar{E}(r) \equiv E(r) / E_{c}$, up to the first order of $\alpha$, the solution to Eq. (53) is approximately given by

$$
\bar{E}(r)=E_{Q}\left(1-\frac{2 \alpha}{45 \pi} E_{Q}^{2}+\cdots\right),
$$

where $E_{Q} \equiv E_{Q}(r) \equiv Q /\left(4 \pi r^{2} E_{c}\right)$. We find that the electric field $E(r)$ is smaller than $Q / 4 \pi r^{2}$, due to the charge screening effect of the vacuum polarization. Substituting this solution (54) into the Einstein equation (52), we obtain the integration

$$
m(r)=M-\int_{r}^{\infty} 4 \pi r^{2} d r \frac{1}{2}\left[E^{2}(r)+\frac{\alpha}{15 \pi} E^{4}(r) / E_{c}^{2}\right] .
$$


This equation clearly shows that the energy-mass function $m(r)$ of Eq. (49) is the total gravitational mass $M$ (attractive) "screened down" by the electromagnetic energy (repulsive). In the Maxwell theory $\left(\Delta \mathscr{L}_{\text {eff }}^{\text {cos }}\right)_{\mathscr{P}}=0$ and $E(r)=Q /\left(4 \pi r^{2}\right)$, we obtain the Reissner-Nordström solution $m(r)=M-Q^{2} / 8 \pi r$. In the Euler-Heisenberg system, it is not proper to make the integration in Eq. [55), since the integrand comes from the Euler-Heisenberg effective Lagrangian, which is valid only for constant fields. In order to gain some physical insight into the energymass function (55), we integrate Eq. (55) to the leading order of $\alpha$,

$$
m(r) \approx M-\frac{Q^{2}}{8 \pi r}\left[1-\frac{\alpha}{225 \pi} \frac{1}{(4 \pi)^{2}} \frac{Q^{2}}{r^{4}} \frac{1}{E_{c}^{2}}\right]=M-\frac{Q^{2}}{8 \pi r}\left[1-\frac{\alpha}{225 \pi} E_{Q}^{2}\right],
$$

which shows the QED correction to the Reissner-Nordström solution. Due to the QED vacuum polarization effect, the black hole charge $Q$ is screened

$$
Q \rightarrow Q\left[1-\frac{\alpha}{225 \pi} E_{Q}^{2}\right]^{1 / 2}
$$

As a consequence, the electrostatic energy of Eq. (56) is smaller than $Q^{2} /(8 \pi r)$ in the ReissnerNordström solution.

Moreover, we study the QED correction to the black hole horizon. For this purpose, we define the horizon radius $r_{H}$ at which the function $f(r)$ of Eq. (49) vanishes, i.e., $f\left(r_{H}\right)=0$, leading to

$$
\frac{G m\left(r_{H}\right)}{r_{H}}=\frac{1}{2} .
$$

Using the energy-mass function $m(r)$ of Eq. (56), we obtain

$$
\frac{G M}{r_{H}}-\frac{G Q^{2}}{8 \pi r_{H}^{2}}\left[1-\frac{\alpha}{225 \pi} E_{Q h}^{2}\right]=\frac{1}{2},
$$

where $E_{Q h} \equiv E_{Q}\left(r_{H}\right)$. Up to the leading order of $\alpha$, we obtain

$$
\begin{aligned}
& r_{H+}=G M+\sqrt{G^{2} M^{2}-\frac{G Q^{2}}{4 \pi}\left[1-\frac{\alpha}{225 \pi} E_{Q+}^{2}\right]}, \\
& r_{H-}=G M-\sqrt{G^{2} M^{2}-\frac{G Q^{2}}{4 \pi}\left[1-\frac{\alpha}{225 \pi} E_{Q-}^{2}\right]},
\end{aligned}
$$

where $E_{Q+} \equiv E_{Q}\left(r_{H_{+}}\right)$and $E_{Q-} \equiv E_{Q}\left(r_{H_{-}}\right)$. Equation (60) shows that the black hole horizon radius $r_{H+}$ becomes larger than the Reissner-Nordström one $r_{+}$given by Eq. (60) for setting $\alpha=0$. The black hole horizon area $4 \pi r_{H+}^{2}$ becomes larger than the Reissner-Nordström one $4 \pi r_{+}^{2}$ given by Eq. (60) for setting $\alpha=0$. This is again due to the black hole charge $Q$ screened by the QED vacuum polarization (57).

In the Reissner-Nordström solution, the extreme black hole solution is given by $r_{+}=r_{-}$or $4 \pi G M^{2}=Q^{2}$. In our case, this is given by $r_{H+}=r_{H-}=r_{H}$ yielding

$$
G^{2} M^{2}-\frac{G Q^{2}}{4 \pi}\left[1-\frac{\alpha}{225 \pi} E_{Q h}^{2}\right]=0 .
$$


From Eqs. (60) and (61), we obtain

$$
\begin{aligned}
4 \pi r_{H}^{2} & =4 \pi G^{2} M^{2}=G Q^{2}\left[1-\frac{\alpha}{225 \pi} E_{Q h}^{2}\right] \\
& =G Q^{2}\left[1-\frac{\alpha}{225 \pi} \frac{1}{G^{2} Q^{2} E_{c}^{2}}\right], \\
r_{H_{ \pm}} & \approx Q\left[1-\frac{\alpha}{225 \pi} E_{Q h}^{2}\right]^{1 / 2}=Q\left[1-\frac{\alpha}{225 \pi} \frac{1}{\left(E_{c} Q\right)^{2}}\right]^{1 / 2} .
\end{aligned}
$$

In Eq. (64) we adopt $G / 4 \pi=1$. Due to the QED correction, the condition of extremely electrically charged black holes with spherical symmetry changes from $M=Q / 4 \pi$ to

$$
M=\frac{Q}{4 \pi}\left[1-\frac{\alpha}{225 \pi} \frac{1}{\left(E_{c} Q\right)^{2}}\right]^{1 / 2} .
$$

This implies that for a given $M$, the black holes are allowed to carry more charge $Q$ than the Reissner-Nordström case. These results show that when the black hole mass $M$ is fixed, the horizon area and radius of the extremely electrically charged black hole are the same as the extreme Reissner-Nordström one. However, when the black hole charge $Q$ is fixed, the black hole horizon area and radius are smaller than those of the extreme Reissner-Nordström black hole. The reason is that the charge screening effect decreases the electrostatic energy; hence, this leads to a smaller mass $M$ for the extreme black hole.

Now we turn to the maximal energy extractable from a black hole. As pointed out in Ref. [62], the surface area $S_{a}$ of the black hole horizon is related to the irreducible mass $M_{i r}$ of the black hole

$$
S_{a}=16 \pi G^{2} M_{i r}^{2}=4 \pi r_{H+}^{2}
$$

where $r_{H+}$ is given by Eq. (60). The surface area of the black hole horizon cannot be decreased by classical processes [62-64]. Any transformation of the black hole which leaves fixed the irreducible mass is called reversible [62,63]. Any transformation of the black hole which increases its irreducible mass, for instance, the capture of a particle with nonzero radial momentum at the horizon, is called irreversible. In irreversible transformations there is always some kinetic energy that is irretrievably lost behind the horizon. Note that transformations which arbitrarily close to reversible ones are the most efficient transformations for extracting energy from a black hole [62, 63]. Following the same argument presented in Ref. [62], and including the leading-order QED correction (56), we obtain the Christodoulou-Ruffini mass formula

$$
M=M_{i r}+\frac{Q^{2}}{16 \pi G M_{i r}}\left[1-\frac{\alpha}{225 \pi} E_{Q+}^{2}\right],
$$

where the electrostatic energy of the black hole is reduced for the reason that the black hole charge is screened down by the QED vacuum polarization effect (57).

The properties of the surface area $S_{a}$ of the black hole horizon and irreducible mass $M_{i r}$ can also been understood from the concepts of information theory [65]. The black hole entropy $S_{\text {en }}$ 
is introduced as the measure of information about a black hole interior which is inaccessible to an exterior observer and is proportional to the surface area $S_{a}$ of the black hole horizon [65]

$$
S_{\text {en }}=S_{a} / 4=\pi r_{H+}^{2}
$$

The physical content of the concept of the black hole entropy derives from the generalized second law of thermodynamics: when common entropy in the black hole exterior plus the black hole entropy never decreases [65]. In the Einstein-Euler-Heisenberg theory, the black hole irreducible mass of Eq. (66) and entropy of Eq. (68) with the QED correction are determined by the horizon radius $r_{H+}$ of Eq. (60) for charged black holes and Eq. (63) for extreme black holes.

Now we consider the physical interpretation of the electromagnetic term in Eq. (67). This term represents the maximal energy extractable from a black hole, which can be obtained by evaluating the conserved Killing integral [38, 66]

$$
\int_{\Sigma_{t}^{+}} \xi_{+}^{\mu} T_{\mu \nu} d \Sigma^{\nu}=4 \pi \int_{r_{H+}}^{\infty} r^{2} T_{0}^{0} d r
$$

where $\Sigma_{t}^{+}$is the spacelike hypersurface in the space-time region that is outside the horizon $r>r_{H+}$ described by the equation $t=$ constant, with $d \Sigma^{v}$ as its surface element vector. $\xi_{+}^{\mu}$ is the static Killing vector field. This electromagnetic term in Eq. (67) is the total energy of the electromagnetic field and includes its own gravitational binding energy. Using the energymomentum tensor of Eq. (51) and weak-field solution (54), we obtain the maximal energy extractable from an electrically charged black hole

$$
\varepsilon_{e x}=\frac{Q^{2}}{8 \pi r_{H+}}\left[1-\frac{\alpha}{225 \pi} E_{Q+}^{2}\right] .
$$

This shows that the black hole maximal extractable energy decreases in comparison with the Reissner-Nordström case $\left(Q^{2} / 8 \pi r_{+}\right)$. This can be explained by the following: (i) the charge screening effect decreases the electrostatic energy; (ii) the black hole horizon radius $r_{H+}$ of Eq. (60) increases, leading to the decrease of the maximally extractable energy, because the most efficient transformations that extract energy from a black hole occur near the horizon. For the extremely electrically charged black hole, the maximally extractable energy is the same as that in the Reissner-Nordström case, when the black hole mass $M$ is fixed; however, it becomes smaller than the Reissner-Nordström one when the black hole electric charge $Q$ is fixed.

\section{Magnetically charged black holes}

Now we turn to study the Einstein-Euler-Heisenberg theory (41) and (47) in the presence of the magnetic field B. As shown by Eq. (6), the magnetic field $\mathbf{B}$ does not contribute to the pairproduction rate so that the process of the electron-positron pair production does not occur for a strong magnetic field B. For this reason, we consider black holes with strong magnetic fields. The conventional black hole with electric and magnetic fields is the rotating charged black hole 
of the Kerr-Newman black hole [67]. However, the solution to a rotating charged black hole in the Einstein-Euler-Heisenberg theory is rather complicated, and we do not consider it in this work. For the sake of simplicity, we study the nonrotating magnetically charged black hole with spherical symmetry in order to investigate the QED corrections in the presence of the magnetic field $\mathbf{B}$ in the Einstein-Euler-Heisenberg theory.

For a nonrotating magnetically charged black hole with magnetic charge $Q_{m}$, the tensor $F_{\mu \nu}$ compatible with spherical symmetry can involve only a radial magnetic field $F_{23}=-F_{32}$. In the Einstein-Maxwell theory, the field equations (28) give (see, e.g., Refs. [68, 69])

$$
F_{23}=\frac{Q_{m} \sin \theta}{4 \pi}
$$

and the gauge potential will be (see, e.g., Refs. [68])

$$
A_{\mu}(x)=\left[0,0,0, Q_{m}(1-\cos \theta) / 4 \pi\right]
$$

The metric is similar to the one of nonrotating electrically charged black holes,

$$
d s^{2}=f(r) d t^{2}-f(r)^{-1} d r^{2}-r^{2} d \Omega, \quad f(r) \equiv 1-2 G m(r) / r
$$

where $m(r)$ is the mass-energy function. In the Einstein-Maxwell theory, the metric function $f(r)$ of magnetically charged black holes with spherical symmetry is given by (see, e.g., Refs. [68])

$$
f(r)=1-\frac{2 G M}{r}+\frac{G Q_{m}^{2}}{4 \pi r^{2}}
$$

where $M$ is the black hole mass seen at infinity.

\subsection{Weak magnetic field case}

Using Eq. (41), we obtain the energy-momentum tensor for the weak magnetic field $B$ case,

$$
T^{\mu v}=T_{M}^{\mu v}\left(1-\frac{2 \alpha B^{2}}{45 \pi E_{c}^{2}}\right)+g^{\mu v} \frac{\alpha B^{4}}{90 \pi E_{c}^{2}}+\cdots .
$$

Similar to the analysis of electrically charged black holes with spherical symmetry, we obtain the (0-0) component of Einstein equations,

$$
\frac{2 m^{\prime}(r)}{r^{2}}=4 \pi\left[B^{2}(r)-\frac{\alpha}{45 \pi} B^{4}(r) / E_{c}^{2}\right] .
$$

For the magnetically charged black hole with spherical symmetry, only a radial magnetic field is present. The field equations (28) give $B(r)=Q_{m} /\left(4 \pi r^{2}\right)$ (see, e.g., Refs. [21,28]). Substituting $B(r)$ into the Einstein equation (76), we obtain the mass-energy function

$$
m(r)=M-\int_{r}^{\infty} 4 \pi r^{2} d r \frac{1}{2}\left[B^{2}(r)-\frac{\alpha}{45 \pi} B^{4}(r) / E_{c}^{2}\right] .
$$


Neglecting the QED correction of the Euler-Heisenberg effective Lagrangian, Eq. (77) gives $m(r)=M-Q_{m}^{2} / 8 \pi r$, which is the solution of the magnetically charged Reissner-Nordström black hole in the Einstein-Maxwell theory. Making the integration in Eq. (77), one obtains [21]

$$
m(r)=M-\frac{Q_{m}^{2}}{8 \pi r}\left[1-\frac{\alpha}{225 \pi} \frac{1}{(4 \pi)^{2}} \frac{Q_{m}^{2}}{r^{4}} \frac{1}{E_{c}^{2}}\right]=M-\frac{Q_{m}^{2}}{8 \pi r}\left[1-\frac{\alpha}{225 \pi} B_{Q}^{2}\right],
$$

where $B_{Q} \equiv B_{Q}(r) \equiv Q_{m} /\left(4 \pi r^{2} E_{c}\right)$. As shown in Eq. (78), taking into account the QED vacuum polarization effect, the total magnetostatic energy is smaller than $Q_{m}^{2} / 8 \pi r$ in the magnetically charged Reissner-Nordström case. This can be understood as follows. In the magnetic field B of the black holes, the vacuum polarization effect results in a positive magnetic polarization $\mathbf{M}$. Then the magnetic $\mathbf{H}$ field defined $\mathbf{B}=\mathbf{H}+\mathbf{M}$ is smaller than the magnetic field $\mathbf{B}$. The magnetostatic energy density $\varepsilon_{E M} \propto \mathbf{B} \cdot \mathbf{H}$ decreases. This shows that in weak magnetic fields, the vacuum polarization effect exhibits the paramagnetic property.

Compared to the result of the electrically charged black hole in the first order of $\alpha$, Eqs. (56) and (78) have the same expression. One can obtain Eq. (78) by simply replacing $E_{Q}$ in Eq. (56) by $B_{Q}$, namely, replacing $Q$ by $Q_{m}$ because of the duality symmetry (see, e.g., Ref. [68]). Similar to the analysis of electric charged black holes, we obtain the horizon radii $r_{H_{+}}$and $r_{H_{-}}$ of the magnetically charged black hole, up to the leading order of $\alpha$,

$$
\begin{aligned}
& r_{H+}=G M+\sqrt{G^{2} M^{2}-\frac{G Q_{m}^{2}}{4 \pi}\left[1-\frac{\alpha}{225 \pi} B_{Q+}^{2}\right]}, \\
& r_{H-}=G M-\sqrt{G^{2} M^{2}-\frac{G Q_{m}^{2}}{4 \pi}\left[1-\frac{\alpha}{225 \pi} B_{Q-}^{2}\right]},
\end{aligned}
$$

where $B_{Q+} \equiv B_{Q}\left(r_{H+}\right)$ and $B_{Q-} \equiv B_{Q}\left(r_{H-}\right)$. The result (79) shows that the black hole horizon radius $r_{H+}$ increases in comparison with the magnetically charged Reissner-Nordström one $r_{+}$. This is again due to the paramagnetic effect of the vacuum polarization that decreases the magnetostatic energy of the black hole.

Now we turn to the extreme black hole $\left(r_{H+}=r_{H-}=r_{H}\right)$. Similarly, we have

$$
G^{2} M^{2}-\frac{G Q_{m}^{2}}{4 \pi}\left[1-\frac{\alpha}{225 \pi} B_{Q h}^{2}\right]=0
$$

where $B_{Q h} \equiv B_{Q}\left(r_{H}\right)$, and we obtain the black hole horizon area and radius

$$
\begin{aligned}
4 \pi r_{H}^{2} & =4 \pi G^{2} M^{2}=G Q_{m}^{2}\left[1-\frac{\alpha}{225 \pi} B_{Q h}^{2}\right]=G Q_{m}^{2}\left[1-\frac{\alpha}{225 \pi} \frac{1}{G^{2} Q_{m}^{2} E_{c}^{2}}\right] \\
r_{H} & \approx Q_{m}\left[1-\frac{\alpha}{225 \pi} B_{Q h}^{2}\right]^{1 / 2}=Q_{m}\left[1-\frac{\alpha}{225 \pi} \frac{1}{\left(E_{c} Q_{m}\right)^{2}}\right]^{1 / 2} .
\end{aligned}
$$

In the second line, we adopt $G / 4 \pi=1$. The QED correction changes the condition of extremely magnetically charged black holes with spherical symmetry from $M=Q_{m} / 4 \pi$ to

$$
M=\frac{Q_{m}}{4 \pi}\left[1-\frac{\alpha}{225 \pi} \frac{1}{\left(E_{c} Q_{m}\right)^{2}}\right]^{1 / 2} .
$$


The properties of the horizon area and radius of the extremely magnetically charged black hole are the same as their counterparts in the extremely electrically charged black hole, given by the duality transformation $Q \leftrightarrow Q_{m}$.

Following the same argument presented in Ref. [62], we obtain the Christodoulou-Ruffini mass formula

$$
M=M_{i r}+\frac{Q_{m}^{2}}{16 \pi G M_{i r}}\left[1-\frac{\alpha}{225 \pi} B_{Q+}^{2}\right]
$$

for magnetically charged black holes with spherical symmetry in the Einstein-Euler-Heisenberg theory. One is able to obtain the irreducible mass $M_{i r}$ by substituting Eq. (79) into Eq. (66), and the black hole entropy $S_{\text {en }}$ by substituting Eq. (79) into Eq. (68). The irreducible mass $M_{i r}$ and the black hole entropy $S_{e n}$ in terms of black hole horizon radius $r_{H+}$ Eq. (79) have the same paramagnetic property in the presence of the QED vacuum polarization effect, as already discussed.

As shown in Eq. (85), the maximal energy extractable from a magnetically charged black hole is

$$
\varepsilon_{e x}=\frac{Q_{m}^{2}}{8 \pi r_{H+}}\left[1-\frac{\alpha}{225 \pi} B_{Q+}^{2}\right],
$$

where $r_{H+}$ is given by Eq. (79). The result shows that the maximal energy extractable from a magnetically charged black hole is smaller than $\frac{Q_{m}^{2}}{8 \pi r_{+}}$of the magnetically charged ReissnerNordström black hole. The reasons are the following: (i) the vacuum polarization effect decreases the magnetostatic energy; (ii) the black hole horizon radius $r_{H+}$ of Eq. (79) increases, therefore the maximally extractable energy decreases. The maximal energy extractable from an extremely magnetically charged black hole is the same as that from an extremely magnetically charged Reissner-Nordström black hole when the black hole mass $M$ is fixed, while it decreases when the black hole magnetic charge $Q_{m}$ is fixed, as we have already discussed at the end of Sec. 4 for the case of the extremely electrically charged black hole.

\subsection{Strong magnetic field case}

In this section, we study the magnetically charged black holes with a strong magnetic field $B(r)$. From Eq. (47), we obtain the energy-momentum tensor of the magnetically charged black hole with spherical symmetry in the strong magnetic field case. Analogous to the weak magnetic field case of magnetically charged black holes with spherical symmetry, we obtain the (0-0) component of Einstein equations

$$
\frac{2 m^{\prime}(r)}{4 \pi r^{2}}=4 \pi\left\{B^{2}(r)+\frac{e^{2} B^{2}}{12 \pi^{2}}\left[\ln \left(\frac{\pi E_{c}}{B}\right)+\gamma-\frac{6}{\pi^{2}} \zeta^{\prime}(2)\right]\right\}
$$


and the field equations (28) give $B(r)=Q_{m} /\left(4 \pi r^{2}\right)$. Substituting this magnetic field $B(r)$ into the Einstein equation (87), we obtain

$$
\begin{aligned}
m(r) & \approx M-\int_{r}^{\infty} 4 \pi r^{2} d r \frac{1}{2}\left\{B^{2}+\frac{e^{2} B^{2}}{12 \pi^{2}}\left[\ln \left(\frac{\pi E_{c}}{B}\right)+\gamma-\frac{6}{\pi^{2}} \zeta^{\prime}(2)\right]\right\} \\
& \approx M-\frac{Q_{m}^{2}}{8 \pi r}\left\{1+\frac{\alpha}{3 \pi}\left[\ln \left(\frac{\pi}{B_{Q}}\right)+\gamma+2-\frac{6}{\pi^{2}} \zeta^{\prime}(2)\right]\right\}
\end{aligned}
$$

This result is valid for $B \gg E_{c}$, for which the value of $\ln \left(\pi / B_{Q}\right)+\gamma+2-\frac{6}{\pi^{2}} \zeta^{\prime}(2)$ is negative. As a result, Eq. (89) shows that the total magnetostatic energy in the presence of the vacuum polarization is smaller than $Q_{m}^{2} / 8 \pi r$ of the magnetically charged Reissner-Nordström black hole. Similar to the weak-field case, this is again due to the paramagnetic effect of the vacuum polarization that decreases the magnetostatic energy of black holes. In the strong magnetic field case, the QED vacuum polarization effect is much larger than the result (78) in the weak-field case, where the QED correction term in Eq. (78) is small for the smallness of $\alpha /(225 \pi)$ and $B_{Q}^{2}$. This result (89) shows a significant QED effect of the vacuum polarization on the energy of magnetically charged black holes in the strong magnetic field case.

Now we turn to the study of the black hole horizon radius and area in the strong magnetic field case. Using the condition $f\left(r_{H}\right)=0$, we obtain the horizon radii $r_{H+}$ and $r_{H-}$ up to the leading order of $\alpha$,

$$
\begin{aligned}
& r_{H+}=G M+\sqrt{G^{2} M^{2}-\frac{G Q_{m}^{2}}{4 \pi}\left[1+\frac{\alpha}{3 \pi} \mathscr{K}_{N R+}\right]}, \\
& r_{H-}=G M-\sqrt{G^{2} M^{2}-\frac{G Q_{m}^{2}}{4 \pi}\left[1+\frac{\alpha}{3 \pi} \mathscr{K}_{N R-}\right]},
\end{aligned}
$$

where

$$
\begin{aligned}
& \mathscr{K}_{N R+}=\ln \left(\frac{\pi}{B_{Q+}}\right)+\gamma+2-\frac{6}{\pi^{2}} \zeta^{\prime}(2), \\
& \mathscr{K}_{N R-}=\ln \left(\frac{\pi}{B_{Q-}}\right)+\gamma+2-\frac{6}{\pi^{2}} \zeta^{\prime}(2) .
\end{aligned}
$$

Equation (90) shows that the horizon radius $r_{H+}$ increases in comparison with the magnetically charged Reissner-Nordström one $r_{+}$. This is again due to the paramagnetic effect of the vacuum polarization that decreases the magnetostatic contribution to the total energy of black holes.

For the case of the extreme black hole $\left(r_{H+}=r_{H-}=r_{H}\right)$, we have

$$
G^{2} M^{2}-\frac{G Q_{m}^{2}}{4 \pi}\left[1+\frac{\alpha}{3 \pi} \mathscr{K}_{N R}\right]=0,
$$

where

$$
\mathscr{K}_{N R}=\ln \left(\frac{\pi}{B_{Q h}}\right)+\gamma+2-\frac{6}{\pi^{2}} \zeta^{\prime}(2) .
$$


As a result, we obtain

$$
\begin{aligned}
4 \pi r_{H}^{2} & =4 \pi G^{2} M^{2}=G Q_{m}^{2}\left[1+\frac{\alpha}{3 \pi} \mathscr{K}_{N R}\right], \\
r_{H} & \approx Q_{m}\left[1+\frac{\alpha}{3 \pi} \mathscr{K}_{N R}\right]^{1 / 2} .
\end{aligned}
$$

Similar to the weak magnetic field case, the QED correction changes the condition of extremely magnetically charged black holes with spherical symmetry from $M=Q_{m} / 4 \pi$ to

$$
M=\frac{Q_{m}}{4 \pi}\left[1+\frac{\alpha}{3 \pi} \mathscr{K}_{N R}\right]^{1 / 2}
$$

These results show that the horizon area and radius of the extreme black hole are the same as their counterparts of the extremely magnetically charged Reissner-Nordström black hole, when the black hole mass $M$ is fixed. Whereas, the black hole magnetic charge $Q_{m}$ is fixed, Eqs. (96) and (97) show that the black hole horizon area and radius become smaller than their counterparts of extremely magnetically charged Reissner-Nordström black holes. We have discussed this behavior in Eqs. (62)- 65) for the case of extremely electrically charged black holes.

Analogously, we obtain the Christodoulou-Ruffini mass formula in the strong-field case of magnetically charged black holes,

$$
M=M_{i r}+\frac{Q^{2}}{16 \pi G M_{i r}}\left[1+\frac{\alpha}{3 \pi} \mathscr{K}_{N R+}\right] .
$$

It is straightforward to obtain irreducible mass $M_{i r}$ by substituting Eq. (90) into Eq. (66), and the black hole entropy $S_{e n}$ by substituting Eq. (90) into Eq. (68). Analogous to the case of the electrically charged black hole, the black hole irreducible mass $M_{i r}$ and entropy $S_{e n}$ in the strong

magnetic field case depend on the black hole horizon radius $r_{H+}$ of Eqs. (90) and (96). Equation (99) indicates that the maximal energy extractable from a magnetically charged black hole is

$$
\varepsilon_{e x}=\frac{Q_{m}^{2}}{8 \pi r_{H+}}\left[1+\frac{\alpha}{3 \pi} \mathscr{K}_{N R+}\right] .
$$

The properties of the maximally extractable energy in the strong magnetic field case are similar to those of the magnetically charged black hole in the weak magnetic field case. However, the QED correction of the vacuum polarization effect to the energy of the magnetically charged black hole in the strong magnetic field case is much more significant in comparison with that in the weak magnetic field case.

\section{Black holes with electric and magnetic charges}

If the spherically symmetric (nonrotating) black hole is both electrically and magnetically charged, electric and magnetic fields do not vanish. As shown in Eq. (11), both invariants $S$ and $P$ contribute to the Euler-Heisenberg effective Lagrangian. The metric takes the same 
form as the metric of Eq. (49) for electrically charged black holes with spherical symmetry. In this case, the tensor $F_{\mu \nu}$ compatible with spherical symmetry can involve only a radial electric field $F_{01}=-F_{10}$ and a radial magnetic field $F_{23}=-F_{32}$, and the gauge potential is (see, e.g., Ref. [68])

$$
A_{\mu}(x)=\left[A(r), 0,0, Q_{m}(1-\cos \theta) / 4 \pi\right] .
$$

In the Einstein-Maxwell theory, $A(r)=-Q /(4 \pi r)$, and the metric function $f(r)$ of Eq. (49) is given by (see, e.g., Ref. [68])

$$
f(r)=1-\frac{2 G M}{r}+\frac{G Q^{2}}{4 \pi r^{2}}+\frac{G Q_{m}^{2}}{4 \pi r^{2}}
$$

In the Einstein-Euler-Heisenberg theory, we study the spherically symmetric black hole with electric and magnetic charges in the weak-field case. Using Eq. (41), we derive the energymomentum tensor with a radial electric field $E$ and a radial magnetic field $B$,

$$
T^{\mu v}=T_{M}^{\mu v}\left[1+\frac{2 \alpha}{45 \pi E_{c}^{2}}\left(E^{2}-B^{2}\right)\right]+g^{\mu v} \frac{\alpha}{90 \pi E_{c}^{2}}\left[\left(E^{2}-B^{2}\right)^{2}+7(E \cdot B)^{2}\right]+\cdots .
$$

Analogous to the analysis of electrically/magnetically charged black holes with spherical symmetry, we obtain the (0-0) component of Einstein equations,

$$
\frac{2 m^{\prime}(r)}{r^{2}}=4 \pi\left[E^{2}(r)+B^{2}(r)+\frac{\alpha}{15 \pi} E^{4}(r) / E_{c}^{2}-\frac{\alpha}{45 \pi} B^{4}(r) / E_{c}^{2}+\frac{\alpha}{9 \pi E_{c}^{2}} E^{2}(r) B^{2}(r)\right] .
$$

In addition, we obtain the field equations from Eq. (28) (see also Ref. [21]),

$$
\begin{aligned}
& E(r)+\frac{2 \alpha}{45 \pi} E^{3}(r) / E_{c}^{2}+\frac{\alpha B^{2}}{9 \pi E_{c}^{2}} E(r)=\frac{Q}{4 \pi r^{2}}, \\
& B(r)=\frac{Q_{m}}{4 \pi r^{2}} .
\end{aligned}
$$

Note that the mixing terms of the electric and magnetic fields in Eqs. (104) and (105) come from the contribution of the invariant $P$. Introducing $\bar{E}(r) \equiv E(r) / E_{c}$, we have

$$
\bar{E}(r)=E_{Q}-\frac{2 \alpha}{45 \pi} E_{Q}^{3}-\frac{\alpha}{9 \pi} B_{Q}^{2} E_{Q}+\cdots
$$

up to the first order of $\alpha$. We substitute the solutions of (106) and (107) into the Einstein equation (104) and obtain the mass-energy function

$$
m(r)=M-\int_{r}^{\infty} 4 \pi r^{2} d r \frac{1}{2} E_{c}^{2}\left[E_{Q}^{2}+B_{Q}^{2}-\frac{\alpha}{45 \pi} E_{Q}^{4}-\frac{\alpha}{45 \pi} B_{Q}^{4}-\frac{\alpha}{9 \pi} B_{Q}^{2} E_{Q}^{2}\right] .
$$

Disregarding the QED correction of the Euler-Heisenberg effective Lagrangian, Eq. (108) gives the solution $m(r)=M-Q^{2} / 8 \pi r-Q_{m}^{2} / 8 \pi r$ for the Reissner-Nordström black hole with electric and magnetic charges. Performing the integration in Eq. (108), we approximately obtain

$$
m(r)=M-\frac{Q^{2}}{8 \pi r}\left[1-\frac{\alpha}{225 \pi} E_{Q}^{2}\right]-\frac{Q_{m}^{2}}{8 \pi r}\left[1-\frac{\alpha}{225 \pi} B_{Q}^{2}\right]+\frac{\alpha}{45 \pi} \frac{Q_{m}^{2}}{8 \pi r} E_{Q}^{2} .
$$


In the limit $Q \gg Q_{m}$, Eq. (109) becomes Eq. (56) of the electrically charged black hole. On the contrary, in the limit $Q_{m} \gg Q$, Eq. (109) becomes Eq. (78) of the magnetically charged black hole. In order to study the effect of the $P$ term in the Euler-Heisenberg effective Lagrangian, we consider the case with large $P$ and small $S$, i.e., $Q_{m} \approx Q$. In this situation, Eq. 109 becomes

$$
m(r)=M-\frac{Q^{2}}{8 \pi r}\left[2-\frac{7 \alpha}{225 \pi} E_{Q}^{2}\right],
$$

for $Q_{m}=Q$, i.e., $S=0$ and large $P$. Comparing to the cases of electrically/magnetically charged black holes, the QED correction to the black hole energy becomes larger, which results from the combination effects of the vacuum polarization on electric and magnetic charges of black holes in the Einstein-Euler-Heisenberg theory.

In the same way that has been discussed in previous sections, up to the leading order of $\alpha$, we obtain the horizon radii $r_{H+}$ and $r_{H-}$ from Eq. (110),

$$
\begin{aligned}
& r_{H+}=G M+\sqrt{G^{2} M^{2}-\frac{G Q^{2}}{4 \pi}\left[2-\frac{7 \alpha}{225 \pi} E_{Q+}^{2}\right]}, \\
& r_{H-}=G M-\sqrt{G^{2} M^{2}-\frac{G Q^{2}}{4 \pi}\left[2-\frac{7 \alpha}{225 \pi} E_{Q-}^{2}\right]},
\end{aligned}
$$

and the Christodoulou-Ruffini mass formula

$$
M=M_{i r}+\frac{Q^{2}}{16 \pi G M_{i r}}\left[2-\frac{7 \alpha}{225 \pi} E_{Q+}^{2}\right]
$$

as well as the maximal energy extractable from a black hole

$$
\varepsilon_{e x}=\frac{Q^{2}}{8 \pi r_{H+}}\left[2-\frac{7 \alpha}{225 \pi} E_{Q+}^{2}\right] .
$$

Analogously, we obtain the irreducible mass $M_{i r}$ by substituting Eq. (111) into Eq. (66), and the black hole entropy $S_{\text {en }}$ by substituting Eq. (111) into Eq. (68). The irreducible mass $M_{i r}$, the black hole entropy $S_{e n}$, and the maximal energy extractable from a black hole receive the same QED correction, but a factor of 7/2 larger, as compared with their counterparts in the case of either electrically or magnetically charged black holes in the weak-field case.

\section{Summary}

In this article, in addition to the Maxwell Lagrangian, we consider the contribution from the QED Euler-Heisenberg effective Lagrangian to formulate the Einstein-Euler-Heisenberg theory. On the basis of this theory, we study the horizon radius, area, total energy, entropy, and irreducible mass as well as the maximally extractable energy of spherically symmetric (nonrotating) black holes with electric and magnetic charges. Our calculations are made up to the leading order of the QED corrections in the limits of strong and weak fields. Our results show 
that the QED correction of the vacuum polarization results in the increase of the black hole horizon area, entropy and irreducible mass, as well as the decrease of the black hole total energy and maximally extractable energy. The reason is that the QED vacuum polarization gives rise to the screening effect on the black hole electric charge and the paramagnetic effect on the black hole magnetic charge. The condition of the extremely charged black hole $M=Q / 4 \pi$ or $M=Q_{m} / 4 \pi$ is modified [ see Eqs. (65), (84), and (98)], which results from the screening and paramagnetic effects.

To end this article, we would like to mention that in the Einstein-Euler-Heisenberg theory, it is worthwhile to study Kerr-Newman black holes, whose electric field $\mathbf{E}$ and magnetic field B are determined by the black hole mass $M$, charge $Q$, and angular momentum $a$ [67]. In addition, it will be interesting to study the QED corrections in black hole physics by taking into account the one-loop photon-graviton amplitudes of the effective Lagrangian (34) [48] and its generalizations [49-53]. We leave these studies for future work.

\section{Acknowledgements}

Yuan-Bin Wu is supported by the Erasmus Mundus Joint Doctorate Program by Grant Number 2011-1640 from the EACEA of the European Commission. We thank the anonymous referee for drawing our attention to the one-loop photon-graviton amplitudes.

\section{References}

[1] M. Born and L. Infeld, Proc. R. Soc. A 144, 425 (1934).

[2] B. Hoffmann, Phys. Rev. 47, 877 (1935).

[3] B. Hoffmann and L. Infeld, Phys. Rev. 51, 765 (1937).

[4] E. S. Fradkin and A. A. Tseytlin, Phys. Lett. B 163, 123 (1985).

[5] A. Abouelsaood, C. G. Callan, C. R. Nappi, and S. A. Yost, Nucl. Phys. B 280, 599 (1987).

[6] A. A. Tseytlin, Nucl. Phys. B 501, 41 (1997).

[7] A. Garcia, H. Salazar, and J. F. Plebanski, Nuovo Cimento B 84, 65 (1984).

[8] M. Demianski, Found. Phys. 16, 187 (1986).

[9] S. Fernando and D. Krug, Gen. Relativ. Gravit. 35, 129 (2003).

[10] T. K. Dey, Phys. Lett. B 595, 484 (2004).

[11] R.-G. Cai, D.-W. Pang, and A. Wang, Phys. Rev. D 70, 124034 (2004).

[12] D. L. Wiltshire, Phys. Rev. D 38, 2445 (1988). 
[13] M. Aiello, R. Ferraro, and G. Giribet, Phys. Rev. D 70, 104014 (2004).

[14] J. Diaz-Alonso and D. Rubiera-Garcia, Phys. Rev. D 81, 064021 (2010).

[15] J. Diaz-Alonso and D. Rubiera-Garcia, Phys. Rev. D 82, 085024 (2010).

[16] J. Diaz-Alonso and D. Rubiera-Garcia, J. Phys. Conf. Ser. 283, 012014 (2011).

[17] J. Diaz-Alonso and D. Rubiera-Garcia, J. Phys. Conf. Ser. 314, 012065 (2011).

[18] H. H. Soleng, Phys. Rev. D 52, 6178 (1995).

[19] H. P. de Oliveira, Classical Quantum Gravity 11, 1469 (1994).

[20] W. Heisenberg and H. Euler, Z. Phys. 98, 714 (1936).

[21] H. Yajima and T. Tamaki, Phys. Rev. D 63, 064007 (2001).

[22] E. Ayón-Beato and A. García, Phys. Rev. Lett. 80, 5056 (1998).

[23] E. Ayón-Beato and A. García, Phys. Lett. B 464, 25 (1999).

[24] D. J. Cirilo Lombardo, Int. J. Theor. Phys. 48, 2267 (2009).

[25] A. Burinskii and S. R. Hildebrandt, Phys. Rev. D 65, 104017 (2002).

[26] I. Dymnikova, Classical Quantum Gravity 21, 4417 (2004).

[27] M. Novello, S. E. Perez Bergliaffa, and J. M. Salim, Classical Quantum Gravity 17, 3821 (2000).

[28] K. A. Bronnikov, Phys. Rev. D 63, 044005 (2001).

[29] M. Hassaïne, and C. Martínez, Phys. Rev. D 75, 027502 (2007).

[30] M. Hassaïne and C. Martínez, Classical Quantum Gravity 25, 195023 (2008).

[31] H. A. González, M. Hassaïne, and C. Martínez, Phys. Rev. D 80, 104008 (2009).

[32] S. H. Mazharimousavi, M. Halilsoy, and O. Gurtug, Classical Quantum Gravity 27, $205022(2010)$.

[33] M. H. Dehghani and H. R. Rastegar Sedehi Phys. Rev. D 74, 124018 (2006).

[34] M. H. Dehghani, S. H. Hendi, A. Sheykhi, and H. Rastegar Sedehi, J. Cosmol. Astropart. Phys. 02 (2007) 020.

[35] S. H. Hendi, Phys. Rev. D 82, 064040 (2010).

[36] J. Schwinger, Phys. Rev. 82, 664 (1951). 
[37] F. Sauter, Z. Phys. 69, 742 (1931).

[38] R. Ruffini, G. Vereshchagin, and S.-S. Xue, Phys. Rep. 487, 1 (2010).

[39] http://www.extreme-light-infrastructure.eu/.

[40] I. S. Gradshteyn, I. M. Ryzhik, in: Table of Integrals, Series and Products, edited by J. Alan (Academic Press, New York, 1994), 5th ed.

[41] R. Ruffini and S.-S. Xue, J. Korean Phys. Soc. 49, S715-S721 (2006).

[42] W. Mielniczuk, J. Phys. A: Math. Gen. 15, 2905 (1982).

[43] S. R. Valluri, D. R. Lamm, and W. J. Mielniczuk, Can. J. Phys. 71, 389 (1993).

[44] Y. M. Cho and D. G. Pak, Phys. Rev. Lett. 86, 1947 (2001).

[45] H. Kleinert, E. Strobel, and S.-S. Xue, Phys. Rev. D. 88, 025049 (2013).

[46] H. Kleinert, Particles and Quantum Fields (2011), section 12, http://users.physik.fu-berlin.de//kleinert/kleiner_reb6/psfiles/index.html.

[47] V. Weisskopf, K. Dan. Vidensk. Selsk. Mat. Fys. Medd. 14, 6 (1936).

[48] I. T. Drummond and S. J. Hathrell, Phys. Rev. D 22, 343 (1980).

[49] P. B. Gilkey, J. Dif. Geom. 10, 601 (1975).

[50] F. Bastianelli, S. Frolov, and A. A. Tseytlin, J. High Energy Phys. 02 (2000) 013.

[51] A. O. Barvinsky and G. A. Vilkovisky, Phys. Rep. 119, 1 (1985); Nucl. Phys. B 333, 471 (1990); Nucl. Phys. B 333, 512 (1990).

[52] Y. V. Gusev, Nucl. Phys. B 807, 566 (2009).

[53] F. Bastianelli, J. M. Dávila, and C. Schubert, J. High Energy Phys. 03 (2009) 086.

[54] J. I. Latorre, P. Pascual, and R. Tarrach, Nucl. Phys. B 437, 60 (1995).

[55] W. Dittrich and H. Gies, Phys. Rev. D 58, 025004 (1998).

[56] G. M. Shore, Nucl. Phys. B 460, 379 (1996).

[57] T. J. Hollowood and G. M. Shore, Phys. Lett. B 655, 67 (2007); Nucl. Phys. B 795, 138 (2008); J. High Energy Phys. 12 (2008) 091.

[58] T. Damour and R. Ruffini, Phys. Rev. Lett. 35, 463 (1975).

[59] G. Preparata, R. Ruffini, and S.-S. Xue, Astron. Astrophys. 338, L87-L90 (1998). 
[60] G. Preparata, R. Ruffini, and S.-S. Xue, J. Korean Phys. Soc. 42, S99-S104 (2003).

[61] R. Ruffini and S.-S. Xue, AIP Conf. Proc. 1059, 72 (2008).

[62] D. Christodoulou and R. Ruffini, Phys. Rev. D 4, 3552 (1971).

[63] D. Christodoulou, Phys. Rev. Lett. 25, 1596 (1970).

[64] S. Hawking, Phys. Rev. Lett. 26, 1344 (1971).

[65] J. D. Bekenstein, Phys. Rev. D 7, 2333 (1973).

[66] R. Ruffini and L. Vitagliano, Phys. Lett. B 545, 233 (2002).

[67] E. T. Newman, E. Couch, K. Chinnapared, A. Exton, A. Prakash, and R. Torrence, J. Math. Phys. 6, 918 (1965).

[68] S. W. Hawking and S. F. Ross, Phys. Rev. D 52, 5865 (1995).

[69] G. W. Gibbons and D. A. Rasheed, Nucl. Phys. B 454, 185 (1995). 